\title{
PMMA Lens Design and Manufacturing for Indoor Uniform Illumination
}

\author{
Dian-Hong LIU ${ }^{1}$, Xiao-Hui ZHANG ${ }^{1^{*}}$ and Chen $\mathrm{CHEN}^{2}$ \\ ${ }^{1}$ Ordnance Engineering Department, Naval University of Engineering, Wuhan 430033, China \\ ${ }^{2}$ Taiyuan Satellite Launch Center, Taiyuan, Shanxi 030000, China \\ *Corresponding author's email:779294469@qq.com
}

Keywords: PMMA lens, Illumination, Manufacturing, CNC

\begin{abstract}
Based on a new ray mapping method in ( $u, v)$ coordinate system, we have designed a polymethyl methacrylate(PMMA) lens for indoor uniform illumination. The lighting area is $18 \mathrm{~m}$ $\times 18 \mathrm{~m}$ and has a distance of $8 \mathrm{~m}$ to the light source. We have calculated the surface curves and lofted them into surface, then the lens three-dimensional model is constructed, the PMMA lens is with a refractive index of 1.4935 , the size of the lens is $38 \mathrm{~mm} \times 38 \mathrm{~mm} \times 18 \mathrm{~mm}$. Since the new ray mapping method applies error control to constructing the lens surface, the surface is discontinuous and consists of 20 surface patches. With the lens model being inserted into the optical simulation software, we can process the lighting simulation by a LED source with 1 million rays. Simulation results show the minimum illuminance on the lighting area is 2.2lux and the average illuminance is about 2.6lux. After constructing arrays with 9 designed lenses, the simulation results show the minimum illuminance on the lighting area is about 21lux, which meets the requirement of indoor illumination. In order to prove the lens model can apply to the indoor lighting situation, we have manufactured a lens with PMMA on precision CNC machine tool. With the manufactured lens, we complete the lighting experiment and obtain the lighting pattern. But we have found dark fringes exist on the lighting pattern and the illuminance around two vertexes are not expected results, that is because the compact discontinuous part of the lens can't be manufactured precisely on precision CNC machine tool, and this bring manufacturing errors to the lens.
\end{abstract}

\section{Introduction}

Due to the degree of design freedom of free-form surface, special attention has been devoted to free-form lens design in recent years. With the outstanding advantages of energy-efficient, LED are ready to finish its historical mission that other light source will be replaced by LED. LED free-form lenses have been designed for different situations [1-5]. At present, many indoor lighting situations use other light source but not LED. There is great demand to design LED lens for indoor uniform illumination.

Based on the new ray mapping method in $(\mathrm{u}, \mathrm{v})$ coordinate system in our previous work [6], we can design a LED free-form lens for large indoor uniform illumination. The lighting area is $18 \mathrm{~m}$ $\times 18 \mathrm{~m}$ and has a distance of $8 \mathrm{~m}$ to the light source. We calculate the surface curves and lofted them into surface, then the lens three-dimensional model is constructed, the surface is discontinuous and consists of 20 surface patches. Simulation results show the minimum illuminance on the lighting area is 2.2lux and the average illuminance is about 2.6lux. After constructing arrays with 9 designed lenses, the simulation results show the minimum illuminance on the lighting area is about 21lux, which meets the requirement of indoor illumination. At last we have manufactured a lens with polymethyl methacrylate on precision CNC machine tool.

\section{PMMA Lens Design}

Our goal is uniform illumination on the indoor lighting area, as shown in Fig.1. The lighting area is $18 \mathrm{~m} \times 18 \mathrm{~m}$ and has a distance of $8 \mathrm{~m}$ to the light source. Through the new ray mapping method in our previous work, we can obtain corresponding points from light source to target plane, then we 
calculate the surface curves and loft them into surface, at last the lens three-dimensional model is constructed, as shown in Fig.2. The material of the lens is polymethyl methacrylate with a refractive index of 1.4935 , the size of the lens is $38 \mathrm{~mm} \times 38 \mathrm{~mm} \times 18 \mathrm{~mm}$. Because the new ray mapping method applies error control to constructing the lens surface, the surface is discontinuous and consists of 20 surface patches.

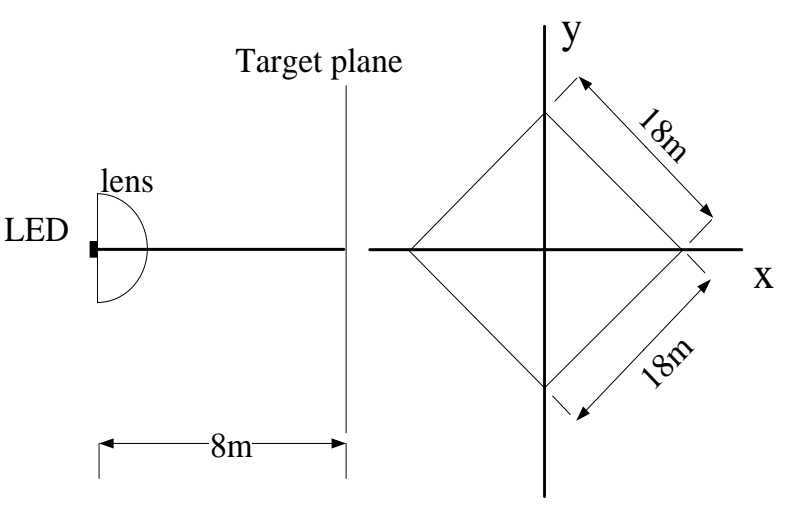

Fig.1. Design parameters

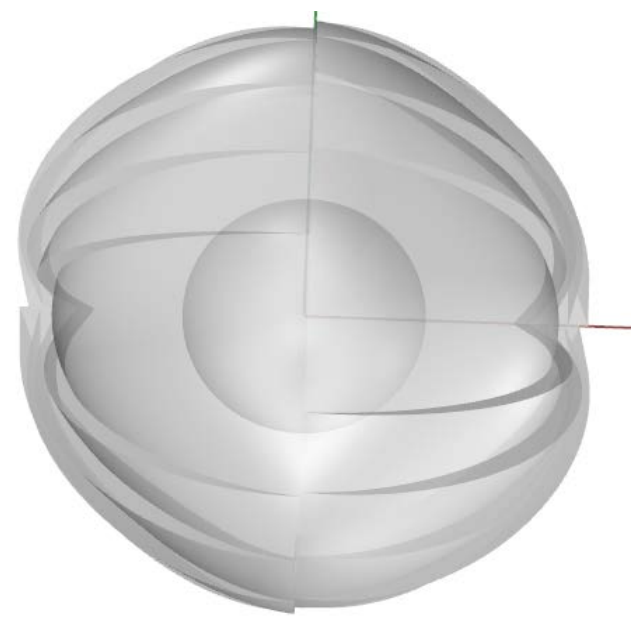

Fig.2. Three-dimensional model of lens

\section{Simulation and Analysis}

We insert the designed lens into the optical simulation software, then process the lighting simulation by a LED source with 1 million rays, as shown in Fig.3. After the simulation, we can obtain the illuminance distribution on the receiving plane, as shown in Fig.4. Simulation results show the minimum illuminance on the lighting area is 2.2lux and the main illuminance on lighting area is about 2.6lux.

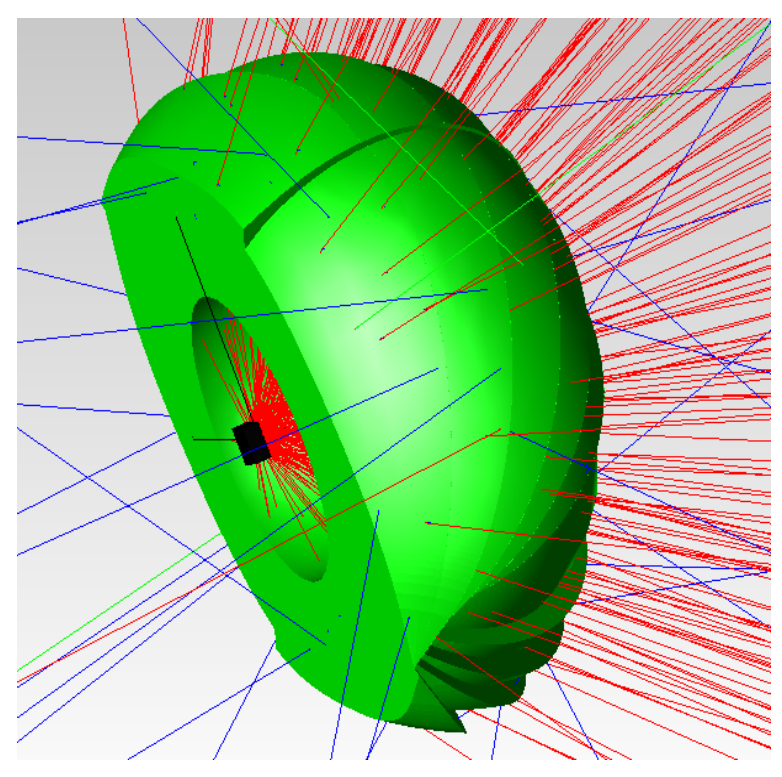

Fig.3. Lighting simulation of the lens
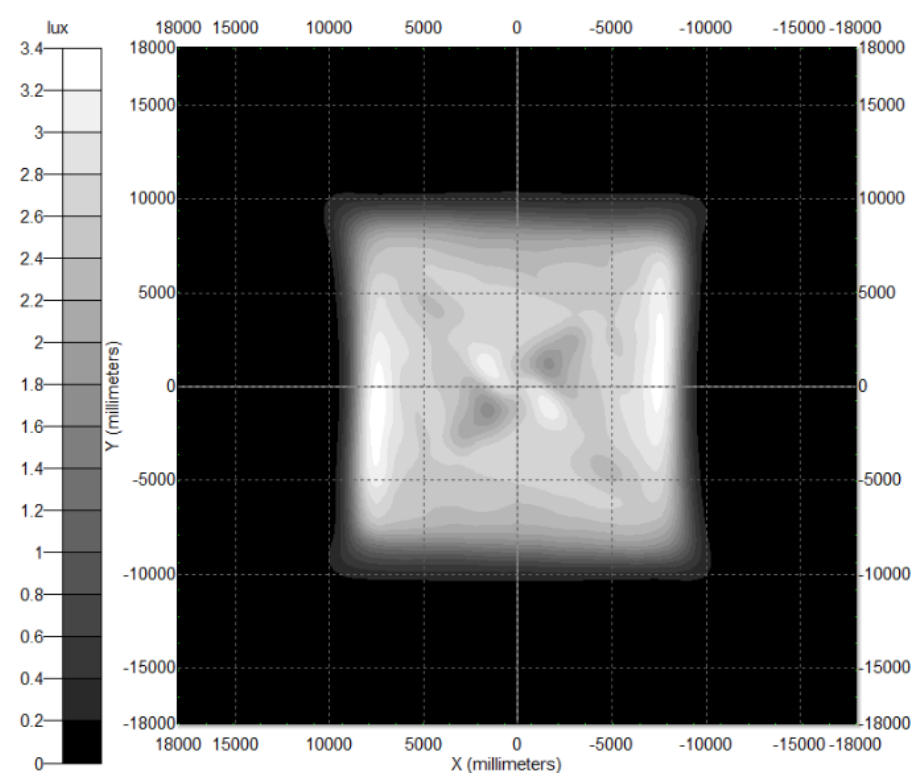

Fig.4. Simulation result of the lens

But the illuminance on the area controlled by one single lens can't satisfy the demand in indoor situation. Considering the small size of the lens, we can construct lenses array by 9 designed lenses, as shown in Fig.5. Since the simulation result in Fig.4 shows two low illuminance areas and two high illuminance areas around the center, we rotate some lenses of the array 90 degrees around z-direction to ensure complementation. After constructing arrays with 9 designed lenses, we process the lighting simulation with 9 LEDs. After the simulation, we can obtain the illuminance distribution on the 
receiving plane, as shown in Fig.4.The simulation results show the minimum illuminance on the lighting area is about 21lux and the main illuminance on lighting area is about 24lux, which meets the

requirement of indoor illumination.

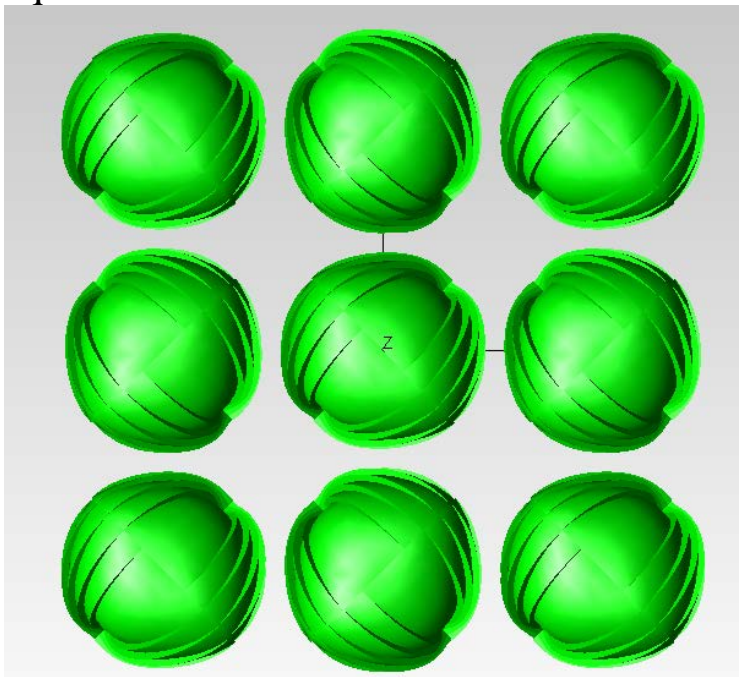

Fig.5. Lighting simulation of the lenses array

\section{Manufacturing and Experimental Analysis}

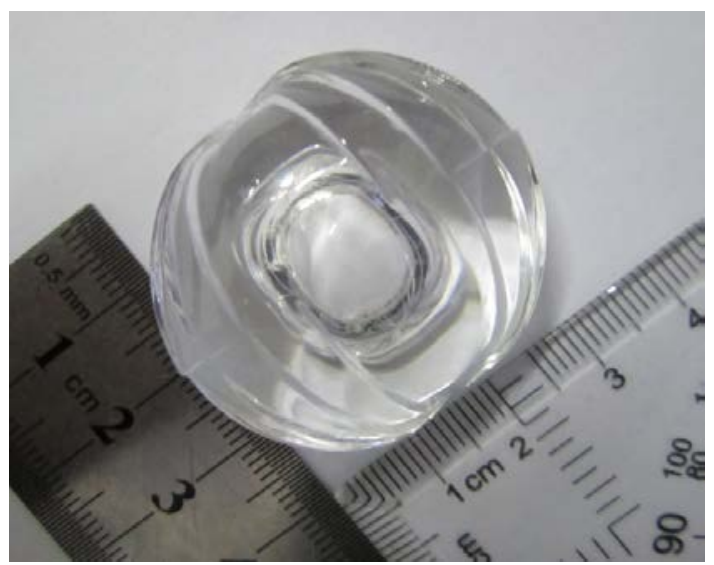

Fig.7. Manufactured lens

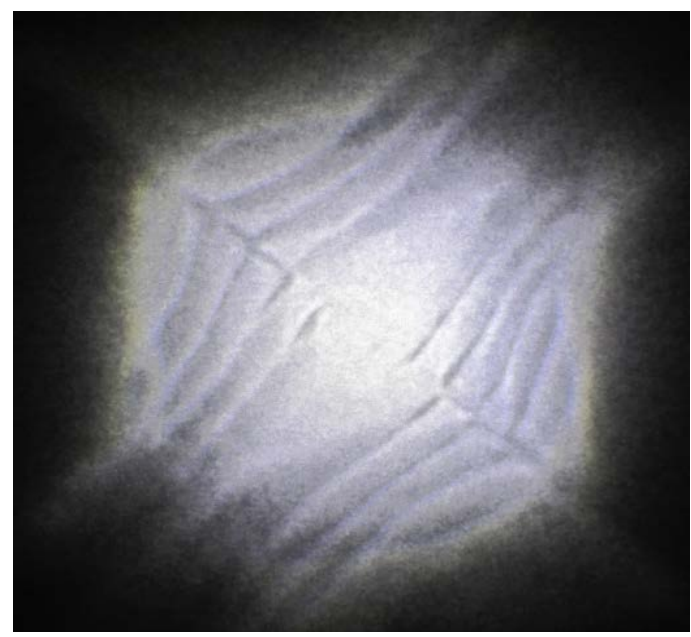

Fig.9. Lighting pattern at a distance of $60 \mathrm{~mm}$

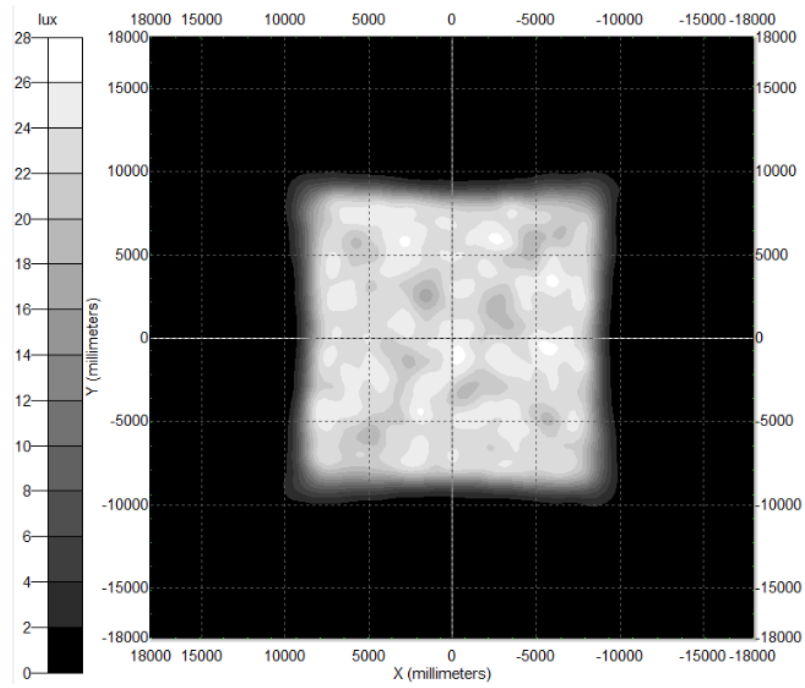

Fig.6. Simulation result of the lenses array

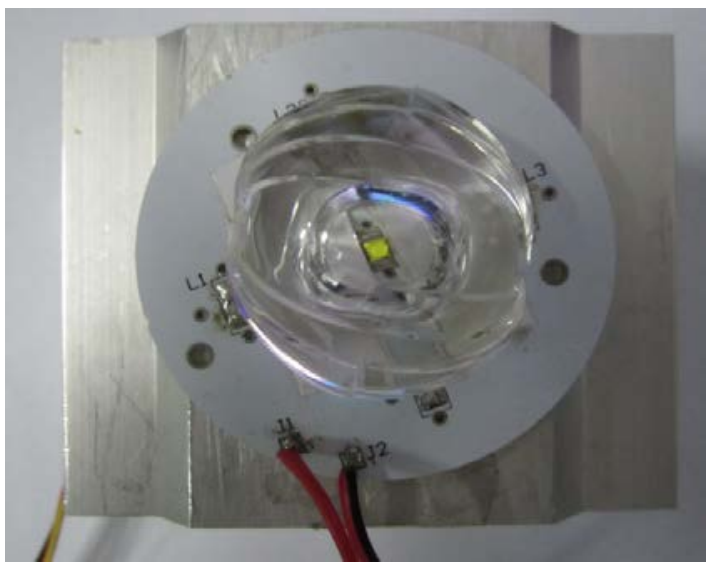

Fig.8. Small platform for the experiment

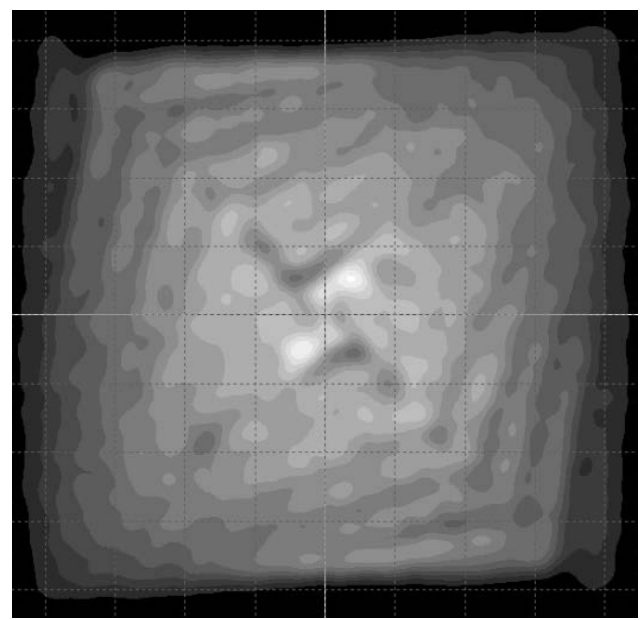

Fig.10. Simulation result at a distance of $60 \mathrm{~mm}$ 
In order to prove the lens model can apply to the indoor lighting situation, we have manufactured the designed lens with polymethyl methacrylate on precision CNC machine tool, the size of the manufactured lens is also $38 \mathrm{~mm} \times 38 \mathrm{~mm} \times 18 \mathrm{~mm}$, as shown in Fig.7. With the manufactured lens, we build a small platform to do the experiment, as shown in Fig.8. After completing the lighting experiment, we obtain the lighting pattern and simulation result at a distance of $60 \mathrm{~mm}$, as shown in Fig.9 and Fig.10. Compared with the simulation result, we have found dark fringes exist on the lighting pattern and the illuminance around two vertexes are not expected results, that is because the compact discontinuous part of the lens can't be manufactured precisely on precision CNC machine tool, and this bring manufacturing errors to the lens, as shown in Fig.11.

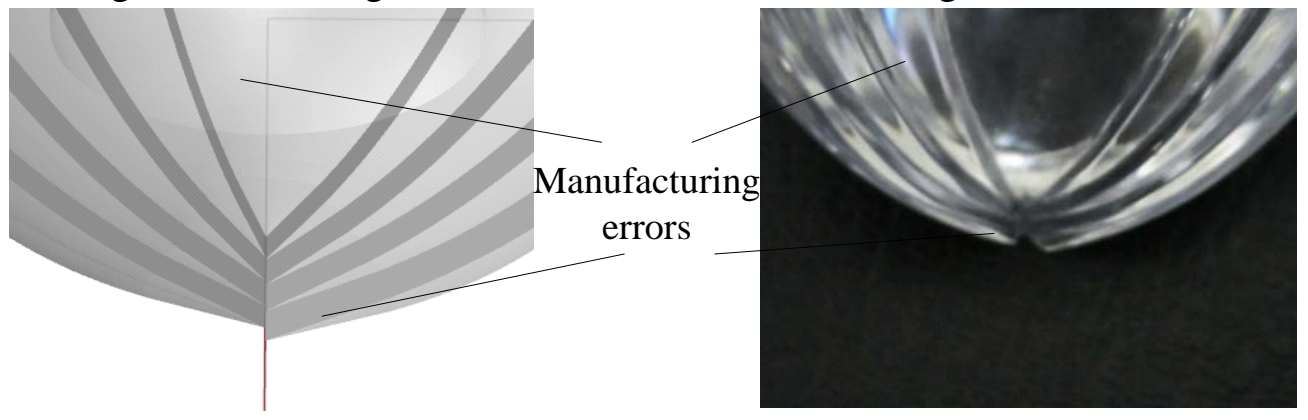

Fig.11. Manufacturing errors of the lens

\section{Conclusion}

In this paper we have designed a LED free-form lens for indoor uniform illumination. The lighting area is $18 \mathrm{~m} \times 18 \mathrm{~m}$ and has a distance of $8 \mathrm{~m}$ to the light source. The lens surface is discontinuous and consists of 20 surface patches. Simulation results of lens show the minimum illuminance on the lighting area is 2.2lux and the average illuminance is about 2.6lux. After constructing arrays with 9 designed lenses, the simulation results show the minimum illuminance on the lighting area is about 21lux, which meets the requirement of indoor illumination. Besides, we have manufactured a lens with polymethyl methacrylate on precision CNC machine tool. With the manufactured lens we complete the lighting experiment. Compared with the simulation result at a distance of $60 \mathrm{~mm}$, we have found dark fringes exist on the lighting pattern and the illuminance around two vertexes are not expected results, that is because the compact discontinuous part of the lens can't be manufactured precisely on precision CNC machine tool, and this bring manufacturing errors to the lens.

\section{References}

[1] Moiseev M A, Doskolovich L L, Kazanskiy N L, Design of high-efficient freeform LED lens for illumination of elongated rectangular regions, Optics Express. 19 (2011) 225-233.

[2] Wu H, Zhang X M, Ge P, A freeform reflector for a light-emitting diode bicycle head lamp, Lighting Research and Technology. 0 (2015)1-17.

[3] Juntunen E, Tetri E, Tapaninen O, A smart LED luminaire for energy savings in pedestrian road lighting, Lighting Research and Technology.47 (2015)103-115.

[4] Moreno I, Avendaño-Alejo M, Saucedo-A T, Modeling LED street lighting, Applied optics. 53 (2014)4420-4430.

[5] Zhenrong Z, Xiang H, Xu L, Freeform surface lens for LED uniform illumination, Applied Optics. 48 (2009)6627-6634.

[6] Dianhong L, Xiaohui Z, Chen C, Free-form lens for rectangular illumination with the target plane rotating at a certain angle, JOSA A. 32 (2015)1958-1963. 